\title{
The Anarchic Braid, Quiescence, Canker- Oral Lichen Planus
}

\section{Anubha B*}

Histopathology and Cytopathology consultant, India

*Corresponding author: Anubha Bajaj, Histopathology and Cytopathology consultant, New Delhi, India, Tel: 00911125117399; Email: anubha.bajaj@gmail.com

\section{Mini Review \\ Volume 3 Issue 1}

Received Date: May 05, 2018

Published Date: May 19, 2018

DOI: $10.23880 /$ cclsj-16000124

\section{Abstract}

Miscellany represents frosted and hyperaemic/ frosted oral mucosal patches. The typical reticular oral lichen planus (OLP) can be distinguished clinically. Lesions elucidating a limited but discriminative morphology or lesions of a specified, definitive aetiology and are equivalent to OLP are alluded to as oral lichenoid reaction or oral lichenoid lesions (OLL's). Diagnostic precision is aspired for, as the management of OLP and OLL"s is contradistinctive. The categorical oral condition is denominated as ISOLATED OLP. When the oral cavity or other mucosal sites demonstrate a paucity of features of the classic Lichen Planus, to arrive at a conclusion of a plaque like or erythematous OLP can be daunting as the histopathology is incapable of segregating the multitudinous variations of the OLL's and OLP. With the paucity of a discernible aetiology, a histological assessment is mandated, chiefly in the non-reticular cases, in order to perceive epithelial dysplasia, carcinoma in situ or invasive squamous cell carcinoma. The label lichenoid dysplasia complicates the terminologies and diagnosis and hence should be withdrawn.

Keywords: Anarchic Braid; Oral Lichen Planus; Quiescence

Abbreviations: OLP: Oral Lichen Planus; OLL's: Oral Lichenoid Lesions.

\section{Introduction}

Miscellany represents frosted and hyperaemic/ frosted oral mucosal patches, the variations of which are clinically detectable such as the geographic tongue and pseudomembranous candidiasis. The typical reticular oral lichen planus (OLP) can be distinguished clinically. Irrefutable, clinically observed oral mucosal OLP lesions elucidating a limited but discriminative morphology or lesions of a specified, definitive aetiology and are equivalent to OLP are alluded to as oral lichenoid reaction or oral lichenoid lesions (OLL's).Thus diagnostic precision is aspired for, as the management of OLP and OLL"s is contradistinctive. Idiopathic OLP is designated for those lesions where the aetiology is lacking. OLP is not an infectious or a contagious disorder. The patho-genetic mechanism of OLP is inadequately described. A minority of cases co-exist with Hepatitis C. A genetic inference is probable. However, more than one family member is uncommonly affected. Immune mechanisms may probably assist with and exhibit clinical and morphological deterioration.

\section{Oral Lichen Planus (OLP)}

Cutaneous lichen planus or LP occurring at an accessory mucosal site is infrequent in patients with definitive OLP. The categorical oral condition is denominated as ISOLATED OLP. A minority of the patients have concurrent ramifications of the skin / alternative mucosal sites such as the vulvovaginal- gingival syndrome 


\section{Cell \& Cellular Life Sciences Journal}

[1]. OLP pemphigoides is singularly uncommon but has been described [2]. OLP preponderates from $0.5 \%$ to $2.2 \%$ in the general population [3]. Conventionally, the spectrum of patient presentation of OLP ranges between 30 to 60 years with a preponderance of females [3].

\section{Clinical Expression}

The lesions are invariably bilateral and the miscellaneous morphologies present proportionately. The reticular, erythematous (erosive), plaque type and ulcerative type are constant and frequent. The atrophic and the bullous variants are sporadic. A single patient may manifest the divergent lesions coincidentally or concurrently. Lichen Planus is frequently displayed on the skin or the mucosal surfaces. These patients appear identical to those manifesting OLP with nonexistent skin /mucosal lesions. The characteristic locales discerned in the OLP are the buccal mucosa, the tongue and the gingiva. Typically, the lesions delineate as a patterned white lace or white and red appliqué forms on the oral mucosa of cheek and tongue. The palate, lips and floor of the mouth are seldom implicated. Burning, itching, stinging discomfort, ulceration and painful sensations are exhibited in the erythematous and ulcerative categories, especially with the consumption of spices, citrus or alcohol. The gingiva is incriminated in DESQUAMATIVE GINGIVITIS where the gums are shiny, red and tender. Lesions of the Reticular Lichen Planus, which are apparent in the oral cavity, are usually deficient in diagnostic characteristics while the non reticular forms are clinically challenging and problematic to diagnose, thus a biopsy is recommended. OLP is a chronic disease of long duration, characterized by multitudinous remissions and exacerbations. The extent of the OLP is determined by the clinical features, designated oral sub-sites and severity of the symptoms [4-6].

\section{Histology and Physiognomy}

The diagnostic paradigm is inspired by the WHO and the details have been specified and derived in 1978 [7]. The histological investigations of OLP are not conclusive per se thus a periodic clinico-pathologic arbitration becomes a pre-requisite. In-spite of the correlation, ambiguous diagnoses may ensue. Immunofluoroscence and Immunohistochemical stains provide a nominal benefit in the diagnosis and classification of OLP [8].

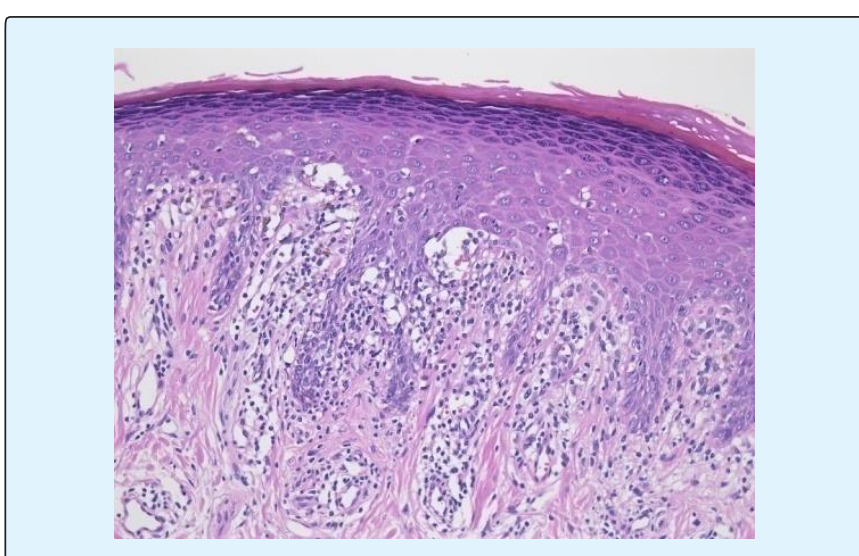

Figure 1: Oral Lichen Planus with saw-toothed rete ridges and band like inflammation in the upper dermis [9].

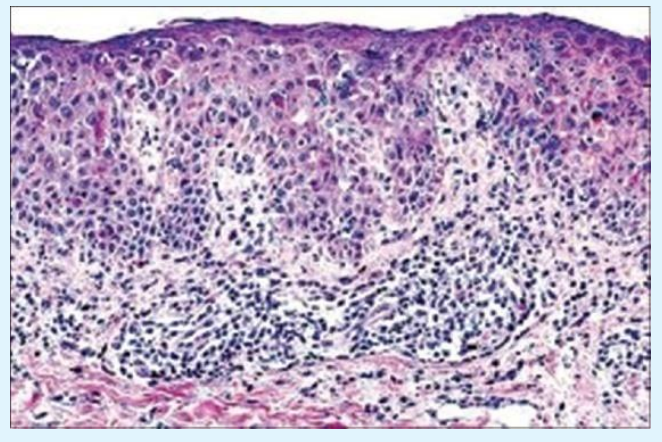

Figure 2: Prominent plasma cell infiltrate in Oral Lichen Planus [10].

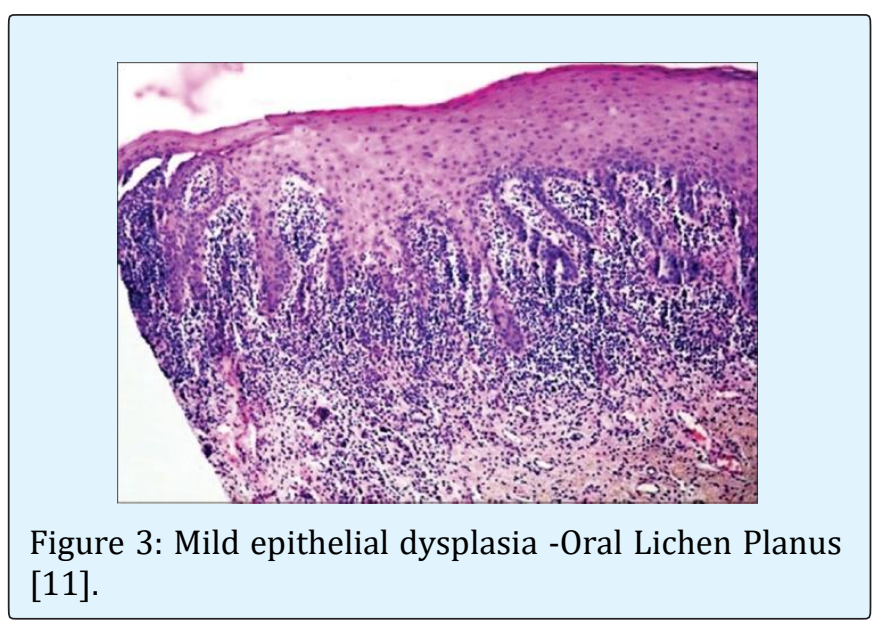




\section{Cell \& Cellular Life Sciences Journal}

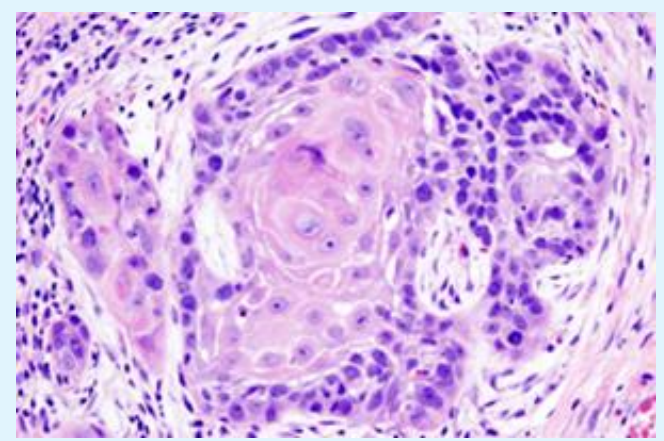

Figure 4: Transformation of Oral Lichen Planus to Oral Squamous Cell Carcinoma [12].
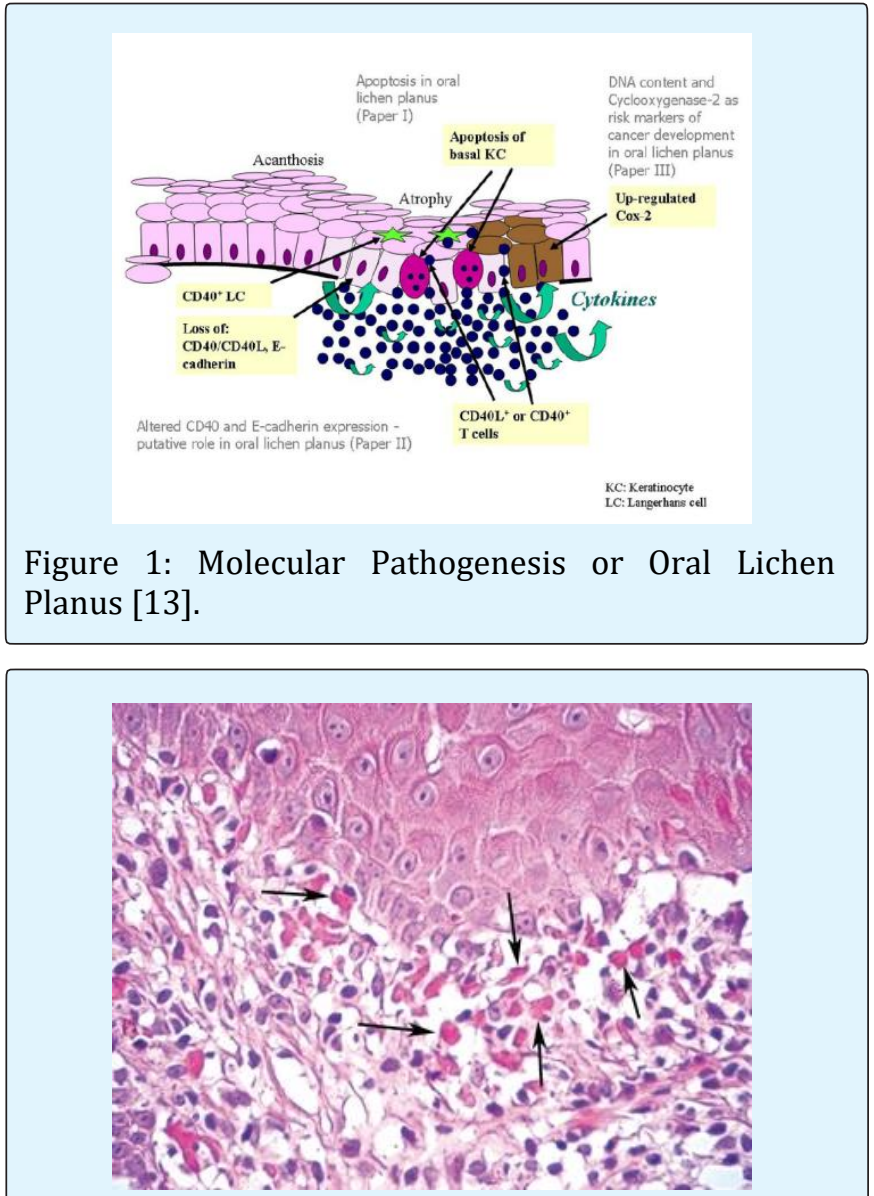

Figure 6: Lichenoid drug reaction with Civatte Bodies [13].

Lichenoid dysplasia generally delineates a lichen planus like histopathology arising in a dysplastic epithelium. In contrast, the inscription invalidates the occurrence of dysplastic alterations in an epithelium affected by LP. The descriptive concept confounds both the pathologists and the clinicians alike.

\section{Therapeutic Modalities}

In established LP only symptomatic therapies are efficacious. Topical medication is requisite for

\section{Oral Lesions}

a) Anaesthetic mouthwashes e.g. Benzydamine

b) Topical steroids as mouthwashes, sprays, pastes and small, dissolvable pellets.

c) In desquamative gingivitis: Oral hygiene is important.

d) Antiseptic oral rinses are necessary as in a daily hydrogen peroxide or bi-weekly chlorhexidine mouthwash.

e) For skin/ scalp/ genital lesions bona fide corticosteroids ointments or creams are administered.

f) Severe circumstances necessitate systemic medication.

g) Surgery or laser treatment is contemplated in persistent and painful lesions

h) Regular haematology/ biochemical profiles and biochemical screens for drug toxicity are required when the individual is on systemic drugs or when therapies such as oral corticosteroids, azathioprine, mycophenolate mofetil etc are in the early phase. There is escalating evidence that the OLP is a low risk, potentially malignant disorder [3]. The annual malignant conversion is proportioned at $0.5 \%$ or less. The entire clinical spectrum with the reticular form can undergo malignant conversion. The evolution of a squamous cell carcinoma is not only confined to the site of genesis of the Oral Lichen Planus, it can emerge from adjacent locations. However, it may be is implausible to be able to predict or prevent the transformation into a neoplasm. The outcome of frequent follow up can be questionable.

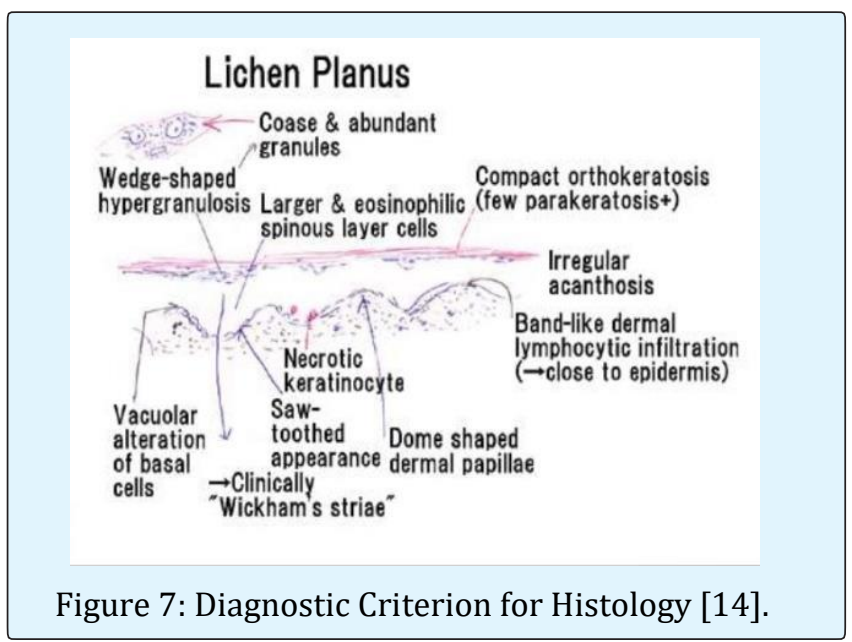




\section{Cell \& Cellular Life Sciences Journal}

\section{Oral Lichenoid Lesions}

Four categories of oral lichenoid lesions (OLL's) can be ascertained which are as follows:

\section{Classification of Oral Lichenoid Lesions (OLL's)}

\begin{tabular}{|c|c|}
\hline 1) & $\begin{array}{c}\text { Amalgam Restoration, Topographically associated } \\
\text { OLL }\end{array}$ \\
\hline 2) & Drug related OLL \\
\hline 3) & OLL in chronic graft versus host disease \\
\hline 4) & $\begin{array}{l}\text { OLL unspecified (e.g. erythematous changes } \\
\text { limited to the gingiva without signs of "classic" OLP } \\
\text { elsewhere in the oral cavity or lesions that have a } \\
\text { lichen planus like aspect but lack one or more of } \\
\text { the characteristic clinical features such as a } \\
\text { bilateral presentation.) }\end{array}$ \\
\hline
\end{tabular}

When the oral cavity or other mucosal sites demonstrate a paucity of features of the classic Lichen Planus, to arrive at a conclusion of a plaque like or erythematous OLP can be daunting. The lichenoid lesions on the mucosal margin of the lip apparently commence due to a microbial plaque originating on the buccal incrust of the anterior teeth.

\section{Clinical Aspects}

Clinical Aspects of the OLL's are identical to the OLP. OLL's may localize or may develop at anomalous oral subsites such as in the patients of chronic graft versus host disease who are known to manifest the lesions of OLP in the palate. With inadequate or insufficient distinct aetiological attributes, the differential diagnosis may incorporate disorders such as erythroleukoplakia. The occurrence of epithelial dysplasia and squamous cell carcinoma and the demarcation between OLP and OLL's usually requires and can be established by a surgical specimen.

\section{Histopathological Evaluation}

Histopathological Evaluation of OLP is incapable of segregating the multitudinous variations of the OLL's and OLP. A mixed sub-epithelial infiltrate is visualized in the OLL's, in contrast to the stringent, dense, band like lymphohistiocytic infiltrate that delineates the OLP. Along with a submerged, diffuse dispersion within the lamina propria and the superficial sub-mucosa are indicators of a drug related lichenoid oral lesion. Focal parakeratosis, focal interruption of the granular layer, cytoid or civatte bodies in the cornified and granular epithelial layers usually typify a lichenoid drug associated reaction. Foci of degeneration of the basement membrane may demonstrate accumulation of granulated mast cells with an enhanced vascular response and an augmented PAS positive basement membrane thickness in OLP as compared to OLL's.

\section{Modulation of the OLL's}

Modulation of The OLL's is achieved by regulation of a component or a measure of a lesion for which an obvious determinant can be established (amalgam, drug related, chronic Graft Versus Host Disease) and is usually based on identifying the aetiology. Amalgam rebuilding and substitution is anatomically linked to the lichenoid, the retrogression of which ensues over several months after cessation of the product. A biopsy is mandatory for patients who elucidate an insufficient reaction to the medication/ therapeutic intervention. Drug related OLL's are infrequent as compared to the cutaneous lichenoid analogues. Persistence of the lesion may be encountered after withdrawal of the offending drug. Oral LP consequents to chronic Graft Versus Host Disease are managed by local corticosteroids or alternative drugs such as tacrolimus. It is complicated to definitely conclude if all OLL's are inherently malignant, particularly amalgam related lichenoid lesions. The accurate procedure is to have the restorations reconstituted for the regression of symptoms and prevention of the unforeseeable evolution of oral cancer. Amalgam restorations, patients with chronic Graft Versus Host Disease and OLL's with non specific clinicopathological delineations may be regarded as a potentially malignant disorder.

\section{Corollaries}

Oral Lichenoid Lesions (OLL's) are those that simulate OLP and are identical on clinical and histological grounds to OLP but enjoy an explicit aetiology. Occasionally the diagnostic precision may not be possible. OLP cannot be conclusively assessed on histopathology alone, since the applicable criterion for histopathology may not be replicated. With the paucity of a discernible aetiology, a histological assessment is mandated, chiefly in the nonreticular cases, in order to perceive epithelial dysplasia, carcinoma in situ or invasive squamous cell carcinoma. The label lichenoid dysplasia complicates the terminologies and diagnosis and hence should be withdrawn. 


\section{Cell \& Cellular Life Sciences Journal}

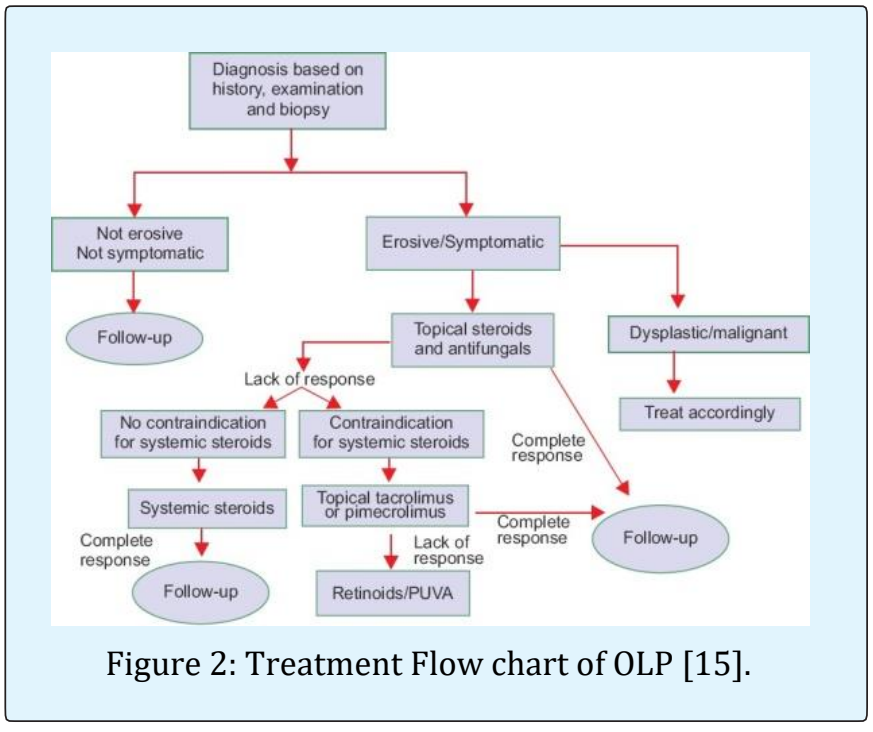

\section{References}

1. Ramer MA, Altchek A, Deligdisch L, Phelps R, Montazem A, et al. (2003) Lichen Planus and the vulvovaginal-gingival syndrome. J Periodontal 74(9): 1385-1393.

2. Solomon LW, Helm TN, Stevens C, Neiders ME, Kumar V (2007) Clinical and Immunopathologic findings in Oral Lichen Planus Pemphigoides. Oral Surg Oral Med Oral Pathol Oral Radiol Endod 103(6): 808-813.

3. Van der Waal I (2009) Oral lichen planus and oral lichenoid lesions; a critical appraisal with emphasis on the diagnostic aspects. Med Oral Patol Oral Cir Bucal 14(7): E310-304.
4. Bethke G, Reichart PA (2005) Assessment of severity of Oral Lichen Planus using a new clinical index. Mund Keifer Gesichtshir 9(3): 152-160.

5. Escudier M, Ahmed N, Shirlaw P, Setterfield J, Tappuni A, et al. (2007) A scoring system for mucosal disease severity with special reference to Oral Lichen Planus. Br J Dermatol 157(4): 765-770.

6. Piboonniyom SO, Treister N, Pitiphat W, Woo SB (2005) Scoring system for monitoring Oral Lichen Planus lesions- a preliminary study. Oral Surg Oral Med Oral Pathol Oral Radiol Endod 99(6): 696-703.

7. Kramer IR, Lucas RB, Pindborg JJ, Sobin LH (1978) Definition of Leukoplakia and related lesions: an aid to studies in oral precancer. Oral Surg Oral Med Oral Pathol 46(4): 518-539.

8. Leaflet on Oral Lichen Planus. British association of Dermatology and British Society of Oral Medicine.

9. Image 1 Courtesy: escholarship.

10. Image 2 Courtesy: International Journal of Health and Allied Sciences. .

11. Image 3 Courtesy: Journal of Oral and Maxillofacial Pathology.

12. Image 4 Courtesy: Union publishing.

13. Image 5 \& 6 Courtesy: Research Gate.

14. Image 7 Courtesy: Slideshare.net.

15. Image 8 Courtesy: Journal of Oral and Maxillofacial Pathology. 